\title{
Dependence of large gradual solar energetic particles on the associated flares and CMEs
}

\author{
Guiming Le *† $^{* \dagger}$ \\ Key Laboratory of Space Weather, National Center for Space Weather, China Meteorological \\ Administration, Beijing, 100081. \\ E-mail: Legmecma.gov.cn

\section{Xuefeng Zhang} \\ School of Computer Science, Anhui University of Technology, Maanshan 243032, Anhui, China. \\ E-mail: zxt Obdahut.edu.cn
}

To investigate the dependence of large gradual solar energetic particle (SEP) events on the associated flares and coronal mass ejections (CMEs), the correlation coefficients (CC) between the peak intensities of $\mathrm{E}>10 \mathrm{MeV}\left(\mathrm{I}_{10}\right), \mathrm{E}>30 \mathrm{MeV}\left(\mathrm{I}_{30}\right)$ and $\mathrm{E}>50 \mathrm{MeV}\left(\mathrm{I}_{50}\right)$ protons and soft X-ray (SXR) emission of associated flares and the speeds of associated CMEs in the three longitudinal areas W0-W39, W40-W70(hereafter well connected region) and W71-W90 have been calculated respectively. The classical correlation analysis shows CCs between SXR emission and the peak intensities of SEP events always reach their largest value in the well connected region and then decline dramatically in the longitudinal area outside the well connected region. For SEP events with source location in the well connected region, the CC between SXR fluence and the peak intensities of SEP events is always larger than the CC between SXR peak flux and the peak intensities of SEP events, suggesting that SXR fluence is a better parameter describing the relationship between flare and SEP events. For SEP events with source location in the well connected region, the $\mathrm{CC}$ between SXR fluence and $\mathrm{I}_{10}$ is $0.58 \pm 0.12$, while the $\mathrm{CC}$ between CME speed and $\mathrm{I}_{10}$ is $0.56 \pm 0.12$. For SEP events with source location in the well connected region, the CC between SXR fluence and $\mathrm{I}_{30}$ is $0.80 \pm 0.06$, while the CC between CME speed and $\mathrm{I}_{30}$ is $0.52 \pm 0.13$. For SEP events with source location in the well connected region, the CC between SXR fluence and $\mathrm{I}_{50}$ is $0.83 \pm 0.06$, while the $\mathrm{CC}$ between $\mathrm{CME}$ speed and $\mathrm{I}_{30}$ is $0.48 \pm 0.13$. The correlation analysis suggests that for SEP events with source location in the well connected region, CME shock is only an effective accelerator for $\mathrm{E}<30 \mathrm{MeV}$ protons, while $\mathrm{E}>30 \mathrm{MeV}$ protons may be mainly accelerated by concurrent flares

35th International Cosmic Ray Conference - ICRC2017

10-20 July, 2017

Bexco, Busan, Korea

* Speaker.

${ }^{\dagger}$ A footnote may follow. 


\section{Introduction}

There are two kinds of solar energetic particle (SEP) events, named as impulsive and gradual SEP events respectively. The former is accompanied by an impulsive flare, while the latter is accompanied with both a gradual flare and a fast coronal mass ejection (CME). Nobody doubts that the solar source of an impulsive SEP event is the associated impulsive flare. However, when a gradual SEP event happened, whether the concurrent flare contributed to the SEP event is still an open question. There were two kinds of point of views on the solar source of gradual SEP events. The first one is that only CME-driven shocks contributes to gradual SEP events (e.g. Reames 1999, Tylka et al. 2005). The second one is that the solar source of a gradual SEP event may be both the concurrent flare and the shock driven by the associated CME (e.g., Kallenrode 2004, Trottet et al. 2015). Cane et al. (2007) suggested that solar flares and CMEs are likely to coexist and the evolution of any event depends on relative importance of the processes. This is also consistent with the statement (Firoz, K. A., et al. 2012) that the type III and type II bursts are successive evolutions and it is difficult to separate them. The investigation of the properties of solar energetic particle events inferred from their associated radio emission suggests that a clear-cut distinction between flare-related and CME-related SEP events is difficult to establish (Kouloumvakos et al. 2015). Some cases' studies (e.g. Miroshnichenko et al., 2005, Aurass et al., 2006, Le et al. 2006, Simnett 2006, Li et al. 2007a, 2007b, 2009, Masson et al. 2009, Grechnev et al. 2008, PerezPeraza et al. 2009, Klein et al. 2014) have shown that relativistic solar protons (RSPs) may be accelerated by the concurrent flares. Some statistical investigations have also been devoted to the study of the relationship between the peak intensities of SEP events and the parameters of the associated solar flares and CMEs (e.g. Dierckxsens et al. 2015, Grechnev et al., 2015, Trottet et al. 2015). However, longitudinal dependence of peak intensities of SEP events with different energies on associated flares have not been investigated in the papers (Dierckxsens et al., 2015, Grechnev et al., 2015, Trottet et al. 2015, Park et al. 2010, 2012).

It has been accepted that the SEPs accelerated by CME-driven shock can be observed in a very large longitudinal area, while flare-accelerated particles can only be observed in a small longitudinal area, especially in the longitudinal area well connected with the source location of associated SEP event. When a solar flare is an eruptive flare, the accompanied CME will open the magnetic field over the associated active region, leading to the flare-accelerated SEPs can escape from the AR and then enter the interplanetary space. Because the magnetic field lines over an AR are very complicated and SEPs not only can propagate along the magnetic field lines, but also can propagate along the direction perpendicular to the magnetic field lines (Bieber et al., 2004, Qin et al., 2007, 2013, 2014, 2015), leading to flare-accelerated particles not only can be observed in the longitudinal area well connected with the SEP source region, but also can be observed in the longitudinal area outside the well connected region. However, the largest flux of flare-accelerated particles can only be observed in the longitudinal area well connected with SEP source location. When a large gradual SEP event happens, if a lot of satellites can be used to observe the SEP flux at every magnetic field line, then it is easy to check whether the SEP flux is longitudinal dependent and reaches its largest flux in the longitudinal area well connected with the SEP source location, which can be used to judge whether associated flares contribute to the production of SEPs in a large gradual SEP event. However, there has been no this kind of SEP data. Statistical correlation analysis can also 
be used to judge whether flares contribute to the production of SEPs. If the peak intensities of SEP events have a good correlation with associated flares with source locations in the well connected region, while the peak intensities of SEP events have a poor correlation with associated flares with source locations outside the well connected region, then we conclude that flares may really contribute to the production of SEPs in large gradual SEP events. The longitudinal dependence of $\mathrm{E} \geq 100 \mathrm{MeV}$ protons on the associated flares and CMEs have been investigated and the results suggest that $\mathrm{E} \geq 100 \mathrm{MeV}$ protons may be mainly accelerated by the associated flares (Le et al., 2017).

How about the situation for protons with different energies. To answer the question, the classical correlation coefficients between peak intensities of protons with different energy and the parameters of associated solar activities for SEP events with source locations in the well connected region and outside the well connected region will be calculated respectively. This is the motivation of the paper. Data sources and definitions are presented in Section 2. The statistical correlation analysis will be given in section 3 . Section 4 is devoted to the summary and discussion.

\section{Data sources and definitions}

A large gradual SEP event, or a solar protons event (SPE), is defined as the proton peak flux is $10 \mathrm{pfu}$ (particle flux unit, particle $\mathrm{cm}^{-2} \mathrm{sr}^{-1} \mathrm{~s}^{-1}$ ) in the $\mathrm{E}>10 \mathrm{MeV}$ channel measured by GOES spacecraft during a solar energetic particle (SEP) event accompanied by both a fast coronal mass ejection and a long duration SXR flare. When a large gradual SEP event happens, the flux of protons with different energy, such as $\mathrm{E}>10 \mathrm{MeV}, \mathrm{E}>30 \mathrm{MeV}$ and $\mathrm{E}>50 \mathrm{MeV}$ protons, may increase at almost the same time, however, the peak fluxes of protons with different energies are different.

The time integral of SXR flux for a flare, fluence $\left(\Phi_{x}\right)$, is defined as

$$
\Phi_{x}=\int_{t_{s}}^{t_{e}}\left[f(t)-f\left(t_{s}\right)\right] d t
$$

where $\mathrm{f}(\mathrm{t})$ is the SXR flux, $t_{s}$ and $t_{e}$ are the start and end time of the SXR flare, respectively. Equation (1) indicates that SXR flux, from which the background has been subtracted, is integrated over the flare start to end times. The flare start, peak and end time has been defined by SEC/NOAA. The flare end time was defined as the time when SXR flux decayed to a middle point between SXR peak flux and background SXR flux (Kubo and Akioka 2004). $\Phi_{x}$ is related to the total energy released by the associated flare (Kubo and Akioka 2004, Chen et al. 2016), indicating that $\Phi_{x}$ is a better parameter describing the properties a SXR emission than SXR peak flux, and SXR fluence has better correlation with peak flux of 15-40 MeV protons than SXR peak flux (Trotte et al., 2015).

The peak intensity of $\mathrm{E}>10 \mathrm{MeV}, \mathrm{E}>30$ and $\mathrm{E}>50 \mathrm{MeV}$ protons observed by the Geostationary Operational Environmental Satellites (GOES) during solar cycle 23 were ever obtained from the website: ( http://spidr.ngdc.noaa.gov/spidr/). For the SPEs that occurred during solar cycle 23, the source location, the CME speed and flare intensity for each SPEs can be directed copied from the paper Cane (2010). The CME speed associated with SPE that occurred on 2005 January 20 used in the paper is $3242 \mathrm{~km} / \mathrm{s}$ (Gopalswamy et al. 2005). The SPEs that occurred during solar cycle 24 are available from http://umbra.nascom.nasa.gov/sdb/goes/particle/. 
The linear speed of a CME, $\mathrm{V}_{C M E}$, can be obtained from the CME catalogue Yashiro et al., 2004, http://cdaw.gsfc.nasa.gov/CME_list/ of Solar and Heliospheric Observatory/Large Angle Spectroscopic Coronagraph (SOHO/LASCO; Brueckneret al., 1995).

The source location that well magnetically connected with the Earth should be located in the west hemisphere of the Sun. 79 SPEs with source location in the west hemisphere that occurred during 1997-2014 were selected.

\section{Classical correlation analysis and results}

Bootstrap method (Wall and Jenkins, 2012) can be used to estimate the statistical uncertainty of the correlation coefficient (Trotte et al., 2015). Because our sample only comprises 79 SPEs, so we also use bootstrap method (Wall and Jenkins, 2012) to estimate the statistical uncertainty of the correlation coefficients. The correlation coefficient was calculated for $\mathrm{N}$ pairs of values chosen at random within the set of $\mathrm{N}$ observations. This procedure was repeated 5000 times.

\subsection{Correlation between SEPs and SXR peak flux}

The source location of flare-accelerated particles are mainly distributed in the longitudinal area ranged from W40 to W70, which can be seen from Figure 2.3 in the paper of Reames (1999). To check whether the correlation coefficient between peak intensities of SEP events and SXR peak flux (also named flare intensity) is longitudinal dependent, the correlation coefficients between SXR peak flux and the peak intensities protons in the three longitudinal areas: W0-W39, W40W70 and W71-W90 have been derived.

The correlation coefficients between $\mathrm{I}_{10}$ and SXR peak flux in the three longitudinal areas W0-W39, W40-W70 and W71-W90 are $0.24 \pm 0.17,0.43 \pm 0.15$ and $0.26 \pm 0.24$ respectively. It is obvious that the correlation coefficient between $\mathrm{I}_{10}$ and the fluence of SXR emission is longitudinal dependent. The largest coefficient coefficient is only $0.43 \pm 0.15$ in the well connected region, suggesting that the peak flux of $\mathrm{E}>10 \mathrm{MeV}$ protons has only a weak correlation with SXR peak fluxes.

The correlation coefficients between $\mathrm{I}_{30}$ and SXR peak flux in the three longitudinal areas W0-W39, W40-W70 and W71-W90 are $0.43 \pm 0.15,0.71 \pm 0.09$ and $0.35 \pm 0.22$ respectively. It is evident that the correlation coefficient between $\mathrm{I}_{30}$ and SXR peak flux is highly longitudinal dependent, and the correlation coefficient between $\mathrm{I}_{30}$ and SXR peak flux reaches its largest value in the well connected region and then declines dramatically for SEP events with source locations outside the well connected region.

The correlation coefficients between $\mathrm{I}_{50}$ and SXR peak flux in the three longitudinal areas W0-W39, W40-W70 and W71-W90 are $0.54 \pm 0.13,0.77 \pm 0.07$ and $0.36 \pm 0.22$ respectively. It is evident that the correlation coefficient between $\mathrm{I}_{50}$ and SXR peak flux is longitudinal dependent, and the correlation coefficient between $\mathrm{I}_{50}$ and SXR peak flux reaches its largest value in the well connected region and then declines dramatically for SEP events with source locations outside the well connected region. 


\subsection{Correlation between SEPs and SXR fluence}

To investigate the relationship between peak intensities of $\mathrm{E}>10 \mathrm{MeV}, \mathrm{E}>30 \mathrm{MeV}$ and $\mathrm{E}>50$ $\mathrm{MeV}$ protons and SXR fluence, the correlation coefficients between SXR fluence and peak intensities of SEP events have been calculated shown in Figure 4 for $\mathrm{E}>10 \mathrm{MeV}$, Figure 5 for $\mathrm{E}>30 \mathrm{MeV}$ and Figure 6 for $\mathrm{E}>50 \mathrm{MeV}$ protons respectively.

We can see from Figure 1 that the correlation coefficients between $\mathrm{I}_{10}$ and the fluence of SXR emission in the three longitudinal areas W0-W39, W40-W70 and W71-W90 are $0.43 \pm 0.15$, $0.58 \pm 0.12$ and $0.39 \pm 0.22$ respectively. It is obvious that the correlation coefficient between $\mathrm{I}_{10}$ and the fluence of SXR emission is longitudinal dependent. The largest coefficient coefficient is $0.58 \pm 0.12$ in the well connected region, suggesting that the peak flux of $\mathrm{E}>10 \mathrm{MeV}$ protons has a moderate correlation with the concurrent flares.

We can see from Figure 2 that the correlation coefficients between $\mathrm{I}_{30}$ and SXR fluence in the three longitudinal areas W0-W39, W40-W70 and W71-W90 are $0.50 \pm 0.14,0.80 \pm 0.06$ and $0.37 \pm 0.22$ respectively. It is evident that the correlation coefficient between $\mathrm{I}_{30}$ and the fluence of SXR emission is highly longitudinal dependent, and $\mathrm{I}_{30}$ has a good correlation with SXR fluence in the well connected region. The correlation coefficient between $\mathrm{I}_{30}$ and SXR fluence declines dramatically for SEP events with source locations outside the well connected region.

We can see from Figure 3 that the correlation coefficients between $I_{50}$ and SXR fluence in the three longitudinal areas W0-W39, W40-W70 and W71-W90 are $0.54 \pm 0.13,0.83 \pm 0.06$ and $0.13 \pm 0.28$ respectively. It is evident that the correlation coefficient between $\mathrm{I}_{5}$ and SXR fluence is highly longitudinal dependent. The correlation coefficient between $\mathrm{I}_{50}$ and SXR fluennce reaches the largest value in the well connected region and then declines dramatically in the longitudinal area outside the well connected region.
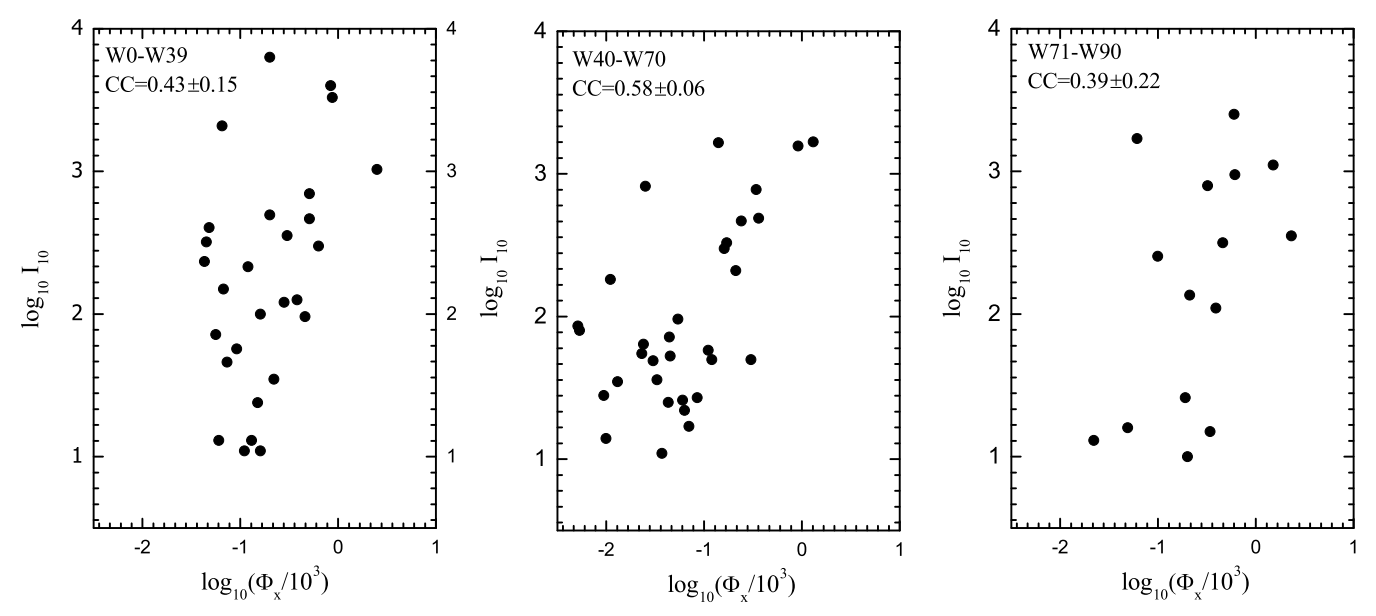

Figure 1: Scatter (log-log) plots of $\mathrm{I}_{10}$ versus $\Phi_{x}$ in the three longitudinal areas respectively.

\subsection{Correlation between peak intensities of SEP events and CME speeds}

To investigate the relationship between CME speeds and the peak intensities of protons with 

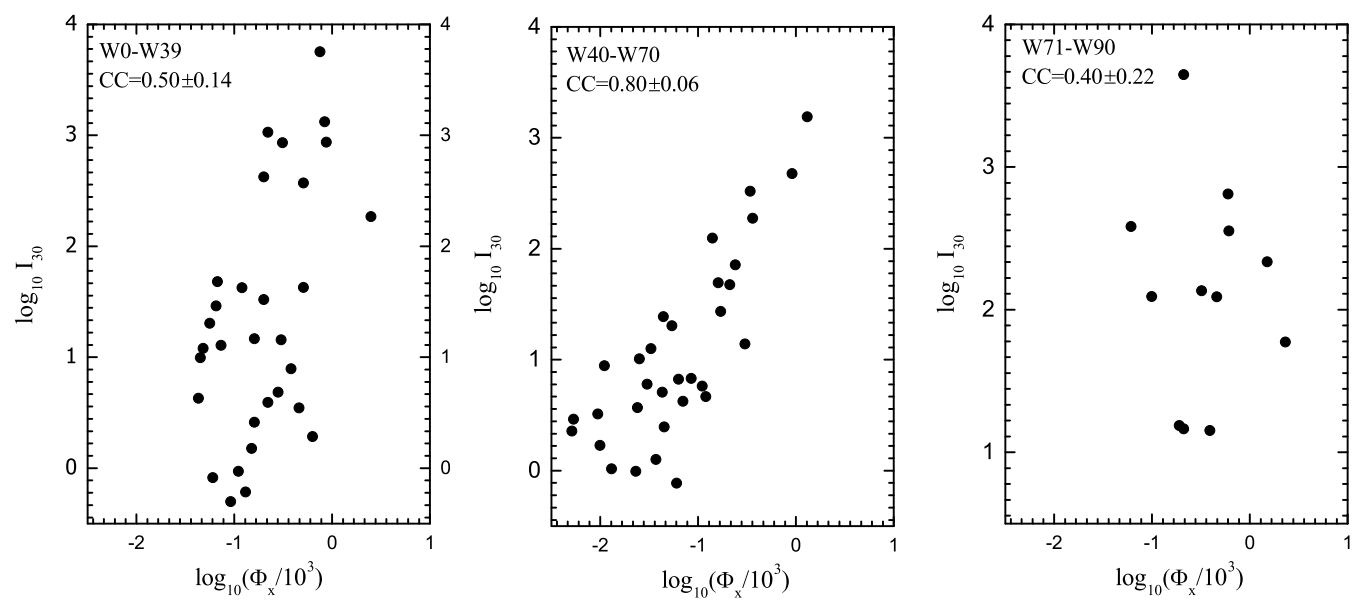

Figure 2: Scatter (log-log) plots of $I_{30}$ versus $\Phi_{x}$ in the three longitudinal areas respectively.
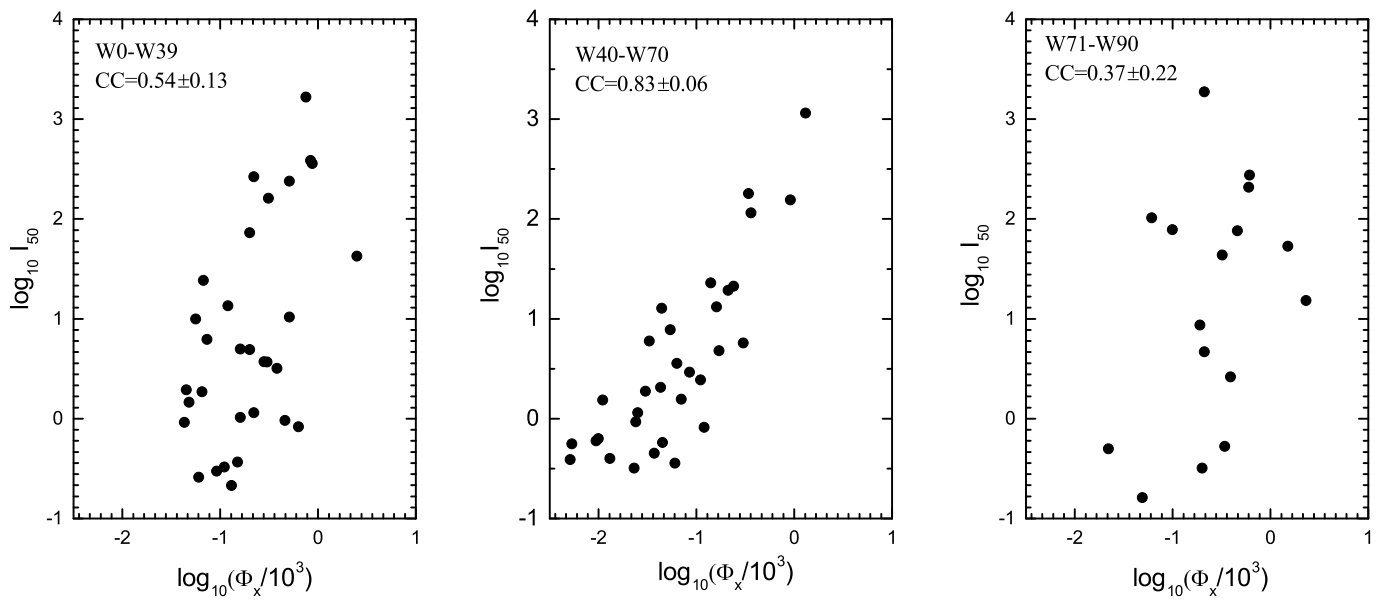

Figure 3: Scatter $(\log -\log )$ plots of $\mathrm{I}_{50}$ versus $\Phi_{x}$ in the three longitudinal areas respectively.

different energies, the correlation coefficients between peak intensities of protons with different energy and CME speeds have been derived and shown in Figures 7 for $\mathrm{E}>10 \mathrm{MeV}$ protons, Figure 8 for $\mathrm{E}>30 \mathrm{MeV}$ protons and Figure 9 for $\mathrm{E}>50 \mathrm{MeV}$ protons respectively.

The correlation coefficient between $\mathrm{I}_{10}$ and CME speeds in the three longitudinal areas W0W39, W40-W70 and W71-W90 are $0.67 \pm 0.10,0.56 \pm 0.12$ and $0.45 \pm 0.21$ respectively.

The correlation coefficient between $\mathrm{I}_{30}$ and CME speeds in the three longitudinal areas W0W39, W40-W70 and W71-W90 are $0.54 \pm 0.10,0.53 \pm 0.13$ and $0.40 \pm 0.21$ respectively.

The correlation coefficient between $\mathrm{I}_{50}$ and CME speeds in the three longitudinal areas W0W39, W40-W70 and W71-W90 are $0.50 \pm 0.14,0.48 \pm 0.14$ and $0.34 \pm 0.23$ respectively. 


\section{Summary and Discussion}

4.1 The classical correlation analysis shows that in the well connected region, SXR fluence contributes to $\mathrm{I}_{10}$, while $\mathrm{E}>30 \mathrm{MeV}$ protons may be mainly accelerated by the concurrent flares. The classical correlation analysis shows that in the well connected region, higher protons have better association with concurrent flares, while lower energy protons have better correlation with the speeds of associated CMEs, suggesting that flares are effective accelerator for higher energy protons, while CME shocks are effective accelerator for lower protons.

4.2 The well connected region may not be exactly in the longitudinal area ranged from W40 to W70. For example, the SPE that occurred on 2003 October 28 with source location at S16E08 was also well connected the Earth at the early phase of the SPE (Miroshnichenko et al. 2005). Anyway, the results of the paper suggest that there is really a longitudinal area within which the correlation coefficient between SXR emission and the peak intensities of SPEs reaches its largest value and then declines for SPEs with source locations outside the well connected region.

\section{References}

[1] Aurass H., Mann, G., Rausche, G., Warmuth, A.(2006), Astron. Astrophys., 457, 681

[2] Bieber J. W., Matthaeus W. H., Shalchi A. and Qin G.(2004), Geophys. Res. Lett., 31, L10805

[3] Brueckner, G.E., Howard, R.A., Koomen, M.J., et al. Solar Phys, 162, 357

[4] Cane, H. V., T. T. von Rosenvinge, C. M. S. Cohen, and R. A. Mewaldt(2003),Geophys. Res. Lett., 30(12), 8017.

[5] Cane, H. V., I. G. Richardson, and T. T. von Rosenvinge (2007), Space Sci. Rev., 130, 301

[6] Cane, H. V., I. G. Richardson, and T. T. von Rosenvinge (2010), J. Geophys. Res., 115, A08101

[7] Chen Yulin, Le Guiming, Yangping Lu, Minhao Chen, Liuguan Ding, Zhiqiang Yin (2016), Astrophys.Space Sci., 361, 40

[8] Dierckxsens M., Tziotziou K., Dalla S., Patsou I., Marsh M.S., Crosby N.B., Malandraki O, Tsiropoula G. (2015), Solar Physics, 290, 841-874

[9] Firoz, K. A., Moon, Y.-J., Park, S.-H., et al. (2011), Astrophys. J., 743, 190 $10.1086 / 341601$

[10] Gopalswamy,N., Xie, H., Yashiro, S.,\&Usoskin, I. (2005), Proc. 29th Int. Cosmic Ray Conf. (Pune), 1, 169

[11] Grechnev, V. V., Kurt, V. G., Chertok, I. M.,?et al.(2008), Sol. Phys., 252, 149

[12] Grechnev, V. V.; Kiselev, V. I.; Meshalkina, N. S.; Chertok, I. M. (2015), Solar Phys, 290, 2827

[13] Kallenrode M-B (2003), J. Phys. G: Nucl. Part. Phys. 29, 965

[14] Klein, K.-L., Trottet, G., Klassen, A. (2010), Solar Phys. , 263, 185

[15] Klein1 K.-L., Masson S., C. Bouratzis, V. Grechnev, A. Hillaris, and P. Preka-Papadema, Astron. Astrophys., A4

[16] Ko, Yuan-Kuen, Tylka, Allan J., Ng, Chee K., et al.(2013), Astrophys. J., 776, 92 
[17] Kouloumvakos A., Nindos A., Valtonen E., Alissandrakis C.E., Malandraki O., Tsitsipis P., Kontogeorgos A., Moussas X., Hillaris A. (2015), Astronomy and Astrophysics, 580, A80,

[18] Kubo, Y., and M. Akioka (2004), Space Weather, 2, S01002

[19] Le Guiming, Yuhua Tang and Yanben Han(2006), ChJAA (Chin. J. of Astron. Astrophys.), 6, 751.

[20] Le, Gui-Ming, Chuan Li, Xue-Feng Zhang (2017), Research in Astron. and Astrophys., 7, 73

[21] Li, C., Tang Y. H., Dai Y., Fang C. and Vial J.C.(2007a), Astron. Astrophys. 472, 283

[22] Li, C., Tang Y. H., Dai Y., Zong, W. G. and Fang C.(2007b), Astron. Astrophys., 461, 1115

[23] Li, C., Dai, Y., Vial, J.C.(2009), Astron. Astrophys., 503, 1013

[24] Li, C., Firoz Kazi A.,Sun L. P.,Miroshnichenko L. I.(2013),Astrophys. J., 770, 11

[25] Li G, Moore R, Mewaldt R A, Zhao L, Labrador A W.(2012), Space Sci. Rev., 171, 141

[26] Masson, S., Klein, K. L., Bütikofer, R., Flückiger, E., Kurt, V., Yushkov, B.,and Krucker, S.(2009),Sol. Phys, 257, 305

[27] McCracken, K. G., H. Moraal, and P. H. Stoker (2008), J. Geophys. Res., 113, A12101

[28] Miroshnichenko, L. I., K.L. Klein, G. Trottet, P. Lantos, E. V. Vashenyuk, Y. V. Balabin, and B. B. Gvozdevsky (2005), J. Geophys. Res., 110, A09S08

[29] Papaioannou, A., Souvatzoglou, G., Paschalis, P. et al. (2014), Sol Phys, 289, 423.

[30] Park, J., Y.J. Moon, D. H. Lee, and S. Youn (2010), J. Geophys. Res., 115, A10105

[31] Park, J., Y.J. Moon, and N. Gopalswamy(2012), J. Geophys. Res., 117, A08108

[32] Perez-Peraza, J., Vashenyuk, E. V., Miroshnichenko, L. I., Balabin, Yu. V., and Gallegos-Cruz, A.(2009), Astrophys. J., 695, 865

[33] Qin G.(2007), Astrophys. J., 656,217

[34] Qin G., Wang Y, Zhang M. and Dalla S.(2013), Astrophys. J., 766,74

[35] Qin G, and Zhang L.-H.(2014), Astrophys. J., 787, 12

[36] Qin G. and Wang Y.(2015), Astrophys. J., 809, 177

[37] Reames, D. V.(1999), Space Sci. Rev., 90, 413

[38] Simnett, G. M., (2006),Astron. Astrophys., 445, 715.

[39] Thakur N., Gopalswamy N, H. Xie, P. M?kel?, S. (2014), Yashiro, S. Akiyama, and J. M. Davila, Astrophys. J., 790, L13

[40] Trottet, G.; Samwel, S.; Klein, K.-L.; Dudok de Wit, T.; Miteva, R. (2015), Solar Physics, 290(3), 819

[41] Tylka, A. J., Cohen, C. M. S., Dierich, W. F., Lee, M. A., Maclennan C.G., Mewaldt, R. A, C. K. Ng, C. K. and D. V. Reames, D. V.,(2005), Astrophys. J., 625, L474

[42] Wall, J.V., Jenkins, C. R. 2012, Cambridge and New York, Chap. 6.6

[43] Yashiro, S., Gopalswamy, N., Michalek, G., St. Cyr, O.C., Plunkett, S.P., Rich, N.B., Howard, R.A. (2004), J. Geophys. Res, 109, A07105 